UT-02-11

\title{
Leptogenesis with Almost Degenerate Majorana Neutrinos
}

\author{
Masaaki Fujii ${ }^{1}$, K. Hamaguchi ${ }^{1}$ and T. Yanagida ${ }^{1,2}$ \\ ${ }^{1}$ Department of Physics, University of Tokyo, Tokyo 113-0033, Japan \\ ${ }^{2}$ Research Center for the Early Universe, University of Tokyo, Tokyo, 113-0033, Japan
}

\begin{abstract}
We investigate the leptogenesis with almost degenerate neutrinos, in the framework of democratic mass matrix, which naturally explains the large mixing angles for neutrino oscillations as well as quark masses and mixing matrix. We find that the baryon asymmetry in the present universe is explained via the decays of righthanded neutrinos produced nonthermally by the inflaton decay. The model predicts neutrinoless double beta decays accessible in near future experiments.
\end{abstract}


One of the fundamental problems in particle physics is to understand the observed masses and mixing angles of quarks and leptons. There have been, in fact, proposed many models of the mass matrices for quarks and leptons. Among them the hypothesis of democratic mass matrix [四] is very interesting not only theoretically, but also phenomenologically. Here, the three families of quarks and leptons are treated in an equal footing, i.e. a permutation symmetry $\mathrm{S}_{3} \times \mathrm{S}_{3}$ is imposed among three families. The democratic mass matrix has been known very successful in explaining the observed masses and mixing angles for quarks [2]. This approach has been extended to the charged lepton and neutrino sectors, and it has been found that the observed large mixings of neutrinos can be rather easily understood due to the nearly diagonal neutrino mass matrix [3], (1. .1] Surprisingly, the model also accounts for the small mixing angle $\mathrm{U}_{\mathrm{e} 3}$, required from the CHOOZ experiment [6], although other two mixing angles are large [0, 8]. Furthermore, this scheme yields almost degenerate Majorana masses for neutrinos ant it predicts neutrinoless double beta decays accessible in near future experiments, such as GENIUS [9], CUORE [10, MOON [11], XMASS [12 and EXO [13.

In this letter, we discuss the leptogenesis [14] in the framework of the democratic mass matrix. We show that the baryon asymmetry in the present universe can be generated via the decay of heavy right-handed neutrinos. Here, the right-handed neutrinos are assumed to be produced nonthermally through inflaton decay [15].

\section{Democratic mass matrix}

Let us start by giving a brief review on the work in Ref. [3], which imposes a permutation symmetry $\mathrm{S}_{3}(L) \times \mathrm{S}_{3}(R)$ on the three families of left-handed lepton doublets $\left(l_{L i}\right)$ and right-handed charged leptons $\left(e_{R i}\right)$. We assume that $l_{L i}$ transform as $\mathbf{3}_{L}=\mathbf{2}_{L}+\mathbf{1}_{L}$ under $\mathrm{S}_{3}(L)$, and $e_{R i}$ transform as $\mathbf{3}_{R}=\mathbf{2}_{R}+\mathbf{1}_{R}$ under $\mathrm{S}_{3}(R)$. Then the mass matrix for charged leptons which is invariant under the $\mathrm{S}_{3}(L) \times \mathrm{S}_{3}(R)$ is uniquely determined as follows:

$$
M_{l}^{(0)}=\frac{\mathcal{M}_{l}}{3}\left[\begin{array}{lll}
1 & 1 & 1 \\
1 & 1 & 1 \\
1 & 1 & 1
\end{array}\right] \text {. }
$$

This mass matrix is often called "democratic" mass matrix [1]. Two mass eigenvalues of this mass matrix vanish. Thus, we introduce a small symmetry-breaking mass term as

\footnotetext{
${ }^{1}$ Ref. [5] considers Dirac or Majorana neutrinos. Thus, their model does not have a group theoretical reason for the family-diagonal and degenerate neutrino masses.
} 
perturbations with a diagonal mass matrix [2]

$$
M_{l}^{(1)}=\mathcal{M}_{l}\left[\begin{array}{ccc}
-\varepsilon_{l} & 0 & 0 \\
0 & \varepsilon_{l} & 0 \\
0 & 0 & \delta_{l}
\end{array}\right]
$$

Then the matrix $M_{l}=M_{l}^{(0)}+M_{l}^{(1)}$ is diagonalized as

$$
V_{l}^{\dagger} M_{l} V_{l}=\operatorname{diag}\left(m_{1}^{l}, m_{2}^{l}, m_{3}^{l}\right),
$$

where

$$
m_{1}^{l}=\mathcal{M}_{l}\left(\frac{\delta_{l}}{3}-\frac{\xi_{l}}{6}\right), \quad m_{2}^{l}=\mathcal{M}_{l}\left(\frac{\delta_{l}}{3}+\frac{\xi_{l}}{6}\right), \quad m_{3}^{l}=\mathcal{M}_{l}\left(1+\frac{\delta_{l}}{3}\right),
$$

with

$$
\xi_{l}=2\left(\delta_{l}^{2}+3 \varepsilon_{l}^{2}\right)^{1 / 2},
$$

where terms with higher order of $\delta_{l}$ and $\varepsilon_{l}$ have been ignored. The unitary matrix $V_{l}$ which diagonalizes the mass matrix $M_{l}$ is given by $V_{l}=A B_{l}$, where

$$
A=\left[\begin{array}{ccc}
1 / \sqrt{2} & 1 / \sqrt{6} & 1 / \sqrt{3} \\
-1 / \sqrt{2} & 1 / \sqrt{6} & 1 / \sqrt{3} \\
0 & -2 / \sqrt{6} & 1 / \sqrt{3}
\end{array}\right]
$$

and

$$
B_{l}=\left[\begin{array}{ccc}
\cos \theta_{l} & -\sin \theta_{l} & -\lambda_{l} \sin 2 \theta_{l} \\
\sin \theta_{l} & \cos \theta_{l} & -\lambda_{l} \cos 2 \theta_{l} \\
\lambda_{l} \sin 3 \theta_{l} & \lambda_{l} \cos 3 \theta_{l} & 1
\end{array}\right], \quad \tan 2 \theta_{l} \simeq \frac{\sqrt{3} \varepsilon_{l}}{\delta_{l}}, \quad \lambda_{l} \simeq \frac{\xi_{l}}{3 \sqrt{2}} .
$$

The empirical masses for the charged leptons $\left(m_{1}^{l}=m_{e}, m_{2}^{l}=m_{\mu}\right.$ and $\left.m_{3}^{l}=m_{\tau}\right)$ are given by adjusting appropriately the parameters $\mathcal{M}_{l}, \varepsilon_{l}$ and $\delta_{l}$ as

$$
\mathcal{M}_{l}=1719[\mathrm{MeV}], \quad \varepsilon_{l}=0.00665, \quad \delta_{l}=0.095,
$$

which leads to $\sin \theta_{l} \simeq 0.066$.

Let us turn to the neutrino sector. Before introducing heavy right-handed neutrinos, we discuss the mass matrix for the light Majorana neutrinos in the effective nonrenormalizable operator:

$$
\frac{f_{i j}}{2 M} l_{L i} H l_{L j} H,
$$


where $H$ denotes the Higgs doublet. There are two independent mass matrices that are invariants under $\mathrm{S}_{3}(L)$ symmetry [3]:

$$
\left[\begin{array}{lll}
1 & 0 & 0 \\
0 & 1 & 0 \\
0 & 0 & 1
\end{array}\right], \quad\left[\begin{array}{lll}
0 & 1 & 1 \\
1 & 0 & 1 \\
1 & 1 & 0
\end{array}\right]
$$

Here we take the first form for the time being, since it automatically leads to the large mixing angles for both of the solar and the atmospheric neutrinos. (We will add the second mass matrix later.) Then, the neutrinos are degenerate in mass. With symmetry breaking terms in diagonal elements, the neutrino mass matrix is given by

$$
M_{\nu}=\frac{f\langle H\rangle^{2}}{M}=\mathcal{M}_{\nu}\left[\begin{array}{lll}
1 & & \\
& 1+\varepsilon_{\nu} & \\
& & 1+\delta_{\nu}
\end{array}\right]
$$

The neutrino-mixing matrix in the basis where the mass matrix for the charged leptons is diagonal is given by $U=\left(V_{l}\right)^{\dagger}$ as 2

$$
U=\left(A B_{l}\right)^{\dagger} \simeq A^{\dagger}=\left[\begin{array}{ccc}
1 / \sqrt{2} & -1 / \sqrt{2} & 0 \\
1 / \sqrt{6} & 1 / \sqrt{6} & -2 / \sqrt{6} \\
1 / \sqrt{3} & 1 / \sqrt{3} & 1 / \sqrt{3}
\end{array}\right]
$$

As denoted, this indicates nearly bi-maximal neutrino oscillations, i.e.,

$$
\sin ^{2} 2 \theta_{12} \simeq 1, \quad \sin ^{2} 2 \theta_{23} \simeq \frac{8}{9}
$$

where $\theta_{12}$ and $\theta_{23}$ are the solar and the atmospheric neutrino mixing angles, respectively. Furthermore, the $\mathrm{U}_{\mathrm{e} 3}$ element automatically vanishes, $\mathrm{U}_{\mathrm{e} 3} \simeq 0$. The mass squared differences for solar and atmospheric neutrino oscillations are given by $\Delta m_{\text {solar }}^{2} \simeq 2 \mathcal{M}_{\nu}^{2} \varepsilon_{\nu}$ and $\Delta m_{\text {atm }}^{2} \simeq 2 \mathcal{M}_{\nu}^{2} \delta_{\nu}$, and hence the breaking parameters should satisfy $\varepsilon_{\nu} / \delta_{\nu} \simeq \Delta m_{\text {solar }}^{2} / \Delta m_{\text {atm }}^{2}$. The experimentally observed values for these quantities are given by [7, 16]

$$
\begin{aligned}
& \Delta m_{\text {atm }}^{2}=\{(1.0-6.0), 3.2\} \times 10^{-3} \mathrm{eV}^{2} \\
& \Delta m_{\text {solar }}^{2}=\{(2-50), 4.9\} \times 10^{-5} \mathrm{eV}^{2} \\
& \sin ^{2} 2 \theta_{23}=\{(0.83-1), 1\} \\
& \tan ^{2} \theta_{12}=\{(0.2-0.8), 0.37\}
\end{aligned}
$$

\footnotetext{
${ }^{2}$ Here, we have neglected the small mixing angle $\theta_{l}$ of charged leptons.
} 
where the last numbers in the parentheses denote the best fit values. Here, we have taken the large mixing MSW (LMA) solution to the solar neutrino problem, which is the most preferable in the present experiments [8].

Recently, a positive indication for neutrinoless double beta decay has been reported, with $\nu_{e}-\nu_{e}$ component of the neutrino mass matrix $\left|m_{\nu_{e} \nu_{e}}\right| \simeq 0.05-0.84 \mathrm{eV}$ [17]. Therefore, it might be interesting to consider the degenerate neutrinos of mass of order $\mathcal{O}(0.1 \mathrm{eV})$. Hereafter, we take $m_{i}^{\nu} \simeq \mathcal{M}_{\nu} \simeq 0.1 \mathrm{eV}$ as a representation. In this case the breaking parameters in the neutrino mass matrix are obtained as $\varepsilon_{\nu}=\Delta m_{\text {solar }}^{2} /\left(2 \mathcal{M}_{\nu}^{2}\right) \simeq 0.001-$ 0.025 and $\delta_{\nu}=\Delta m_{\mathrm{atm}}^{2} /\left(2 \mathcal{M}_{\nu}^{2}\right) \simeq 0.05-0.3$. We see that their orders of magnitudes are just around those of the breaking parameters in charged lepton mass matrix, $\varepsilon_{l}=0.00665$ and $\delta_{l}=0.095$, in Eq. (8).

One may worry about that the predicted value of the mixing angle for the solar neutrino $\theta_{12}$ in Eq. (13) is too large to fit the experimental value. Even if we include the effect of nonzero $\sin \theta_{l} \simeq 0.066$, the mixing angle of the solar neutrino oscillation is only slightly reduced to $\sin ^{2} 2 \theta_{12} \simeq 0.99$. However, a deviation from the nearly maximal mixing for solar neutrino oscillation can be easily implemented by introducing small off-diagonal elements $\kappa_{\nu}$ in the neutrino mass matrix Eq (11):

$$
M_{\nu}=\mathcal{M}_{\nu}\left[\begin{array}{ccc}
1 & \kappa_{\nu} & \kappa_{\nu} \\
\kappa_{\nu} & 1+\varepsilon_{\nu} & \kappa_{\nu} \\
\kappa_{\nu} & \kappa_{\nu} & 1+\delta_{\nu}
\end{array}\right]
$$

Notice that the matrix of the off-diagonal elements has the form of the second matrix in Eq. (10), which is also invariant under the $S_{3}(L)$ as noted before. The mixing matrix of neutrino-oscillations is then given by $U=\left(V_{l}\right)^{\dagger} V_{\nu}$. Here, $V_{\nu}$ is defined as

$$
V_{\nu}^{\dagger} M_{\nu} V_{\nu}=\operatorname{diag}\left(m_{1}^{\nu}, m_{2}^{\nu}, m_{3}^{\nu}\right)
$$

where $m_{1}^{\nu} \simeq \mathcal{M}_{\nu}, m_{2}^{\nu} \simeq \mathcal{M}_{\nu}\left(1+\varepsilon_{\nu}\right), m_{3}^{\nu} \simeq \mathcal{M}_{\nu}\left(1+\delta_{\nu}\right)$. Here and hereafter, we require $\kappa_{\nu}<\varepsilon_{\nu}$ in order to ensure the nearly bi-large mixing for neutrino oscillations.

The neutrino mass matrix given in Eq. (15) has another interesting prediction on the size of the $\mathrm{U}_{e 3}$. When the neutrino mass matrix is diagonal as in Eq. (11), the neutrino mixing matrix $\mathrm{U}$ is entirely determined by $V_{l}$ given in Eqs. (6) and (7). In this case, we can immediately obtain the $\mathrm{U}_{e 3}$ element as

$$
\mathrm{U}_{e 3}=-\frac{2}{\sqrt{6}} \sin \theta_{l}+\frac{\lambda_{l}}{\sqrt{3}} \sin 3 \theta_{l} \simeq-0.05 \text {. }
$$


In the case of the general neutrino mass matrix, the above prediction on the $\mathrm{U}_{e 3}$ is shifted by the non-zero elements of the $V_{\nu}$. The leading deviation of the $\mathrm{U}_{e 3}$ from the value presented in Eq. (17) is given by

$$
\Delta \mathrm{U}_{e 3} \simeq \frac{\cos \theta_{l}}{\sqrt{2}}\left(V_{\nu 13}-V_{\nu 23}\right)
$$

We now consider the specific case where the neutrino mass matrix is given by Eq. (15). One might naively imagine that the deviation of the $\mathrm{U}_{e 3}$ is given by

$$
\Delta \mathrm{U}_{e 3}=\mathcal{O}\left(\frac{\kappa_{\nu}}{\delta_{\nu}}\right) \sim 10^{-(1-2)}
$$

from the above general argument. If this is true, we can have no precise prediction on the size of the $\mathrm{U}_{e 3}$. Interestingly, however, this is not the case. Because of the symmetric form of the neutrino mass matrix in Eq. (15), the leading contribution given in Eq. (18) beautifully cancel out. Actually, the leading terms in $V_{\nu 13}$ and $V_{\nu 23}$ are given by

$$
\begin{aligned}
& V_{\nu 13}=\frac{\kappa_{\nu}}{\delta_{\nu}}, \\
& V_{\nu 23}=\frac{\kappa_{\nu}}{\delta_{\nu}}+\frac{\varepsilon_{\nu} \kappa_{\nu}}{\delta_{\nu}^{2}} .
\end{aligned}
$$

Hence, the deviation of the $\mathrm{U}_{e 3}$ in this model is highly suppressed, $\Delta \mathrm{U}_{e 3} \lesssim 10^{-3}$, which allows us to have a precise prediction, $\left|\mathrm{U}_{e 3}\right| \simeq 0.05 .3$ This will be clearly shown in the numerical calculations presented in this letter. In the future, this prediction on the size of the $\mathrm{U}_{e 3}$ will be tested in the long baseline experiments, such as JHF 18].

The most natural way to explain small neutrino masses is the "see-saw" mechanism [19]. In this case, the mass matrix $M_{\nu}$ for the light neutrinos is given by

$$
M_{\nu}=m_{\nu D}^{T} M_{R}^{-1} m_{\nu D}
$$

after integrating out the heavy right-handed Majorana neutrinos $N_{R i}$. Here, $M_{R}$ and $m_{\nu D}$ are the mass matrices of the right-handed Majorana neutrinos $N_{R i}$ and the Dirac mass for $l_{L i}$ and $N_{R j}$, respectively. In terms of the Yukawa couplings, the Dirac neutrino mass matrix is given by $\left(m_{\nu D}\right)_{i j}=\left(h_{D}\right)_{i j}\langle H\rangle$, where $\left(h_{D}\right)_{i j}$ is defined as $\mathcal{L}=\left(h_{D}\right)_{i j} N_{R i} l_{L j} H$. The $\mathrm{S}_{3}$ invariant matrices of $M_{R}$ and $h_{D}$ are uniquely determined as

$$
M_{R}=\mathcal{M}_{R}\left[\begin{array}{ccc}
1 & \kappa_{R} & \kappa_{R} \\
\kappa_{R} & 1 & \kappa_{R} \\
\kappa_{R} & \kappa_{R} & 1
\end{array}\right], \quad h_{D}=k_{D}\left[\begin{array}{ccc}
1 & \kappa_{D} & \kappa_{D} \\
\kappa_{D} & 1 & \kappa_{D} \\
\kappa_{D} & \kappa_{D} & 1
\end{array}\right]
$$

\footnotetext{
${ }^{3}$ In fact, $V_{\nu 13}=V_{\nu 23}$ in the limit of the vanishing $\varepsilon_{\nu}$.
} 
where we have assumed that the $N_{R i}$ transform as $\mathbf{3}_{L}=\mathbf{2}_{L}+\mathbf{1}_{L}$ under the $\mathrm{S}_{3}(L)$ symmetry. . One can easily imagine that the assumption of $\kappa_{R} \sim \kappa_{D}\left(\sim \kappa_{\nu}\right)$ for the off-diagonal elements is the simplest and natural way to obtain the mass matrix for the light neutrinos in Eq (15) with $\varepsilon_{\nu}=\delta_{\nu}=0$. In order to induce the symmetry breaking parameters $\varepsilon_{\nu}$ and $\delta_{\nu}$ in the neutrino mass matrix in Eq. (15), we introduce breaking parameters $\varepsilon_{D}$ and $\delta_{D}$ in diagonal elements of Yukawa matrix $h_{D}$, as in charged leptons:

$$
h_{D}=k_{D}\left[\begin{array}{ccc}
1 & \kappa_{D} & \kappa_{D} \\
\kappa_{D} & 1+\varepsilon_{D} & \kappa_{D} \\
\kappa_{D} & \kappa_{D} & 1+\delta_{D}
\end{array}\right] \text {. }
$$

Then, we can obtain the required mass matrix of the light neutrinos by taking the following pattern of the perturbations:

$$
\kappa_{R} \sim \kappa_{D}\left(\sim \kappa_{\nu}\right)<\varepsilon_{D}\left(\sim \varepsilon_{\nu}\right) \sim \mathcal{O}(0.01)<\delta_{D}\left(\sim \delta_{\nu}\right) \sim \mathcal{O}(0.1)
$$

The above breakings of the $\mathrm{S}_{3}$ symmetries might be understood as follows. Suppose that the $\mathrm{S}_{3}$ breakings are originated only from the Yukawa coupling of Higgs field $H$, as in mass matrices for quarks and charged leptons. Then, the $\mathrm{S}_{3}(L)$ symmetry is broken in the neutrino Dirac Yukawa matrix by the parameters $\varepsilon_{D}$ and $\delta_{D}$ as in charged lepton sector, since it comes from the coupling of Higgs field. On the other hand, the mass matrix of the right-handed neutrinos $M_{R}$ in Eq. (22) is assumed to be $\mathrm{S}_{3}$ invariant since it is decoupled from the Higgs field $H$.

The mass matrix $M_{R}$ for the heavy right-handed neutrino leads to two exactly degenerate right-handed neutrinos and a slightly heavier/lighter one, with masses $M_{R i}=$ $\left\{\mathcal{M}_{R}\left(1-\kappa_{R}\right), \mathcal{M}_{R}\left(1-\kappa_{R}\right), \mathcal{M}_{R}\left(1+2 \kappa_{R}\right)\right\}$. As we will see in the remainder of this letter, the off-diagonal elements $\kappa_{R}$ and $\kappa_{D}$, which are required to explain the neutrino oscillation experiments, play a crucial role in the leptogenesis.

\footnotetext{
${ }^{4}$ If $N_{R i}$ transform as $\mathbf{3}_{R}=\mathbf{2}_{R}+\mathbf{1}_{R}$ under the $\mathrm{S}_{3}(R)$, the Dirac Yukawa matrix becomes democratic one as in Eq. (1), which invalidates the almost diagonal mass matrix for light neutrinos in Eq. (15).
} 


\section{leptogenesis [14]}

Now let us discuss the leptogenesis in the present model. Notice that the conventional leptogenesis scenario where the right-handed neutrinos are produced by thermal scatterings after the inflation is somewhat difficult in the case of the degenerate neutrinos of $m_{1}^{\nu} \simeq m_{2}^{\nu} \simeq m_{3}^{\nu} \simeq \mathcal{O}(0.1) \mathrm{eV}$. This is because the out-of-equilibrium condition cannot be satisfied in this case and the amount of produced lepton asymmetry is strongly suppressed [20]. (See Appendix.) Therefore, we consider the leptogenesis via decays of the right-handed neutrinos $N_{R i}$ which are produced non-thermally. A natural mechanism of such a non-thermal production of $N_{R i}$ is the decays of the inflaton $\varphi$ into the $N_{R i}$ [15]. Hereafter, we will consider the supersymmetry (SUSY) theory, although the following discussion does not change much in the non-supersymmetric case.

If $C P$ is not conserved in the Yukawa matrix $h_{D}$, the interference between decay amplitudes of tree and one-loop diagrams results in the lepton-number production [14]. The lepton-number asymmetry per a decay of right-handed neutrino $N_{R i}$ is given by 14 , 21]

$$
\begin{aligned}
\epsilon_{i} & \equiv \frac{\sum_{j} \Gamma\left(N_{R i} \rightarrow l_{L j}+H\right)-\sum_{j} \Gamma\left(N_{R i} \rightarrow \overline{l_{L j}}+\bar{H}\right)}{\sum_{j} \Gamma\left(N_{R i} \rightarrow l_{L j}+H\right)+\sum_{j} \Gamma\left(N_{R i} \rightarrow \overline{l_{L j}}+\bar{H}\right)} \\
& =-\frac{1}{8 \pi} \frac{1}{\left(h_{D} h_{D}^{\dagger}\right)_{i i}} \sum_{k \neq i} \operatorname{Im}\left[\left\{\left(h_{D} h_{D}^{\dagger}\right)_{i k}\right\}^{2}\right]\left[\mathcal{F}_{V}\left(\frac{M_{k}^{2}}{M_{i}^{2}}\right)+\mathcal{F}_{S}\left(\frac{M_{k}^{2}}{M_{i}^{2}}\right)\right]
\end{aligned}
$$

where $N_{R i}, l_{L j}$, and $H\left(\overline{l_{L j}}\right.$ and $\left.\bar{H}\right)$ symbolically denote fermionic or scalar components of corresponding supermultiplets (and their anti-particles), and $\mathcal{F}_{V}(x)$ and $\mathcal{F}_{S}(x)$ represent the contributions from vertex and self-energy diagrams, respectively. In the case of the SUSY theory, they are given by [22]

$$
\mathcal{F}_{V}(x)=\sqrt{x} \ln \left(1+\frac{1}{x}\right), \quad \mathcal{F}_{S}(x)=\frac{2 \sqrt{x}}{x-1} .
$$

Here, we have assumed that the mass difference of the right-handed neutrinos is large enough compared with their decay widths, so that the perturbative calculation is ensured. (We will justify this assumption later.)

In the present model, the masses $M_{R i}$ are almost degenerate, $M_{R i}=\left\{\mathcal{M}_{R}(1-\right.$ $\left.\left.\kappa_{R}\right), \mathcal{M}_{R}\left(1-\kappa_{R}\right), \mathcal{M}_{R}\left(1+2 \kappa_{R}\right)\right\}$. Thus, $x \simeq 1$ and the self-energy contribution $\mathcal{F}_{S}(x)$ is much larger than the vertex contribution $\mathcal{F}_{V}(x)$. In the leading order in perturbation,

\footnotetext{
${ }^{5}$ If we take into account the effect of the finite decay width, $\mathcal{F}_{S}(x)$ vanishes for $x \rightarrow 1$. Thus, $\mathcal{F}_{S}\left(M_{k}^{2} / M_{i}^{2}\right)$ in Eq. (25) vanish for $i=1, k=2$ and $i=2, k=1$. See also footnote 6 .
} 
the asymmetry parameters are given by

$$
\epsilon_{1}=\frac{1}{12 \pi} k_{D}^{2} \frac{\operatorname{Im}\left(\kappa_{D}\right)}{\operatorname{Re}\left(\kappa_{R}\right)} \varepsilon_{D}^{2}, \quad \epsilon_{2}=\frac{1}{9 \pi} k_{D}^{2} \frac{\operatorname{Im}\left(\kappa_{D}\right)}{\operatorname{Re}\left(\kappa_{R}\right)} \delta_{D}^{2}, \quad \epsilon_{3}=\epsilon_{1}+\epsilon_{2},
$$

where we have neglected higher order terms in the expansions of $\varepsilon_{D}, \delta_{D}, \kappa_{D}, \kappa_{R}$ and $\varepsilon_{D} / \delta_{D}$. As for the $C P$ phase, we have assumed that the complex phase exists only in the off-diagonal elements $\kappa_{D}$ and $\kappa_{R}$ for simplicity, and have taken the other parameters to be real. Notice that all the decays of $N_{R i}$ generate the lepton asymmetry with the same sign, namely, they contribute in a constructive way.

The ratio of the lepton number density $n_{L}$ to the entropy density $s$ produced by the inflaton decay is given by [15]

$$
\frac{n_{L}}{s}=\frac{3}{2} \sum_{i} \epsilon_{i} B_{r}^{(i)} \frac{T_{R}}{m_{\phi}}
$$

where $T_{R}$ is the reheating temperature after the inflation, $m_{\phi}$ the mass of the inflaton, and $B_{r}^{(i)}$ the branching ratio of the decay channel of the inflaton to $N_{R i}$, i.e., $B_{r}^{(i)}=B_{r}\left(\phi \rightarrow N_{R i} N_{R i}\right)$. Here, we have assumed that the inflaton decays into a pair of right-handed neutrinos, and $M_{R i}>T_{R}$ in order to make the generated lepton asymmetry not washed out by lepton-number violating processes after the $N_{R i}$ 's decay. Notice that the inflaton mass $m_{\phi}$ should satisfy $m_{\phi}>2 \mathcal{M}_{R}$ in order to make the decay $\left(\phi \rightarrow N_{R i} N_{R i}\right)$ kinematically allowed.] After being produced, a part of the lepton asymmetry is immediately converted [14] into the baryon asymmetry via the "sphaleron" effect [24], since the decays of $N_{R i}$ take place much before the electroweak phase transition:

$$
\frac{n_{B}}{s}=C \frac{n_{L}}{s},
$$

where $C$ is given by $C \simeq-0.35$ in the minimal SUSY standard model (MSSM) 25.].

Therefore, the amount of the baryon asymmetry in the present model is estimated as

$$
\frac{n_{B}}{s} \simeq 0.3 \times 10^{-10} \times\left(B_{r}^{(2)}+B_{r}^{(3)}\right)\left(\frac{T_{R}}{10^{8} \mathrm{GeV}}\right)\left(\frac{2 \mathcal{M}_{R}}{m_{\phi}}\right)\left(\frac{\mathcal{M}_{\nu}}{0.1 \mathrm{eV}}\right)\left(\frac{\delta_{D}}{0.1}\right)^{2} \frac{\operatorname{Im}\left(\kappa_{D}\right)}{\operatorname{Re}\left(\kappa_{R}\right)},
$$

\footnotetext{
${ }^{6}$ One might wonder if this fact might conflict with the argument that the generated lepton asymmetry must vanish in the limit of exactly degenerate masses. However, if the mass differences of the right-handed neutrinos become smaller than the decay widths of them, the perturbative formula $\mathcal{F}_{S}(x)$ in Eq. (26) no longer holds and we should take into account the effect of finite widths of $N_{R i}$. Actually, it was shown that $\mathcal{F}_{S}(x)$ vanishes in the limit of exactly degenerate right-handed neutrino masses if the finite decay widths are appropriately taken into account [23].

${ }^{7}$ In this letter, we assume a perturbative decay of the inflaton.
} 
where we have used the see-saw formula $\mathcal{M}_{\nu} \simeq k_{D}^{2}\langle H\rangle^{2} / \mathcal{M}_{R}$. We see that the empirical baryon asymmetry $n_{B} / s \simeq(0.4-1) \times 10^{-10}[26$ is obtained with a reheating temperature $T_{R} \simeq 10^{8}-10^{10} \mathrm{GeV}$, for a natural choice of the parameters, say, $B_{r}^{(2)}+B_{r}^{(3)} \simeq 0.1-1$, $2 \mathcal{M}_{R} \simeq(0.1-1) m_{\phi}, \delta_{D} \simeq 0.1$ and $\operatorname{Im}\left(\kappa_{D}\right) / \operatorname{Re}\left(\kappa_{R}\right) \simeq 1$

Notice that the mass differences of the right-handed neutrinos, $\Delta M_{R} \simeq 3 \operatorname{Re}\left(\kappa_{R}\right) \mathcal{M}_{R}$ is much larger than the decay widths of them, $\Gamma_{N_{R i}} \simeq\left(k_{D}^{2} / 4 \pi\right) \mathcal{M}_{R}$, as long as $\kappa_{R} \gg$ $k_{D}^{2} / 12 \pi \simeq 10^{-6} \times\left(\mathcal{M}_{\nu} / 0.1 \mathrm{eV}\right)\left(\mathcal{M}_{R} / 10^{10} \mathrm{GeV}\right)$, so that the perturbative formula $\mathcal{F}_{S}(x)$ in Eq. (26) is justified. The assumption $M_{R i}>T_{R}$ is also easily satisfied for $M_{R i}>10^{10} \mathrm{GeV}$.

In Fig. 1, we show the histograms of the $n_{B} / s,\left|\mathrm{U}_{e 3}\right|$ and $\mathcal{M}_{\nu}$ for the LMA solution. Here, we randomly generate the small perturbations as in Eq. (24) and collect the data set of $n_{B} / s,\left|\mathrm{U}_{e 3}\right|$ and $\mathcal{M}_{\nu}$ if the generated mass spectrum and mixing angles satisfy the conditions obtained from the neutrino oscillation experiments given in Eq. (14). Then we plot the frequencies of these quantities in the vertical axes. We also present a plot of the obtained mass differences and mixing angles for the solar neutrino oscillation in $\left\{\tan ^{2} \theta_{12}, \Delta m_{\text {solar }}^{2}\right\}$ plane. We take the range of the perturbations as follows:

$$
\begin{array}{ll}
\delta_{D}=(0.25-1.5) \times 10^{-1}, & \varepsilon_{D}=\delta_{D} 10^{-(0.3-2.5)}, \\
\kappa_{D}=\varepsilon_{D} 10^{-(0-0.7)} e^{i(0-2) \pi}, & \kappa_{R}=\left|\kappa_{D}\right| e^{i\left(-\frac{1}{2} \sim \frac{1}{2}\right) \pi},
\end{array}
$$

where we take $\varepsilon_{D}$ and $\delta_{D}$ to be real, for simplicity. If we randomly generate the perturbations within the above range, the neutrino mass spectrum and mixing angles satisfy the conditions given in Eq. (14) with the rate of nearly $20 \%$. The baryon asymmetry $n_{B} / s$ is calculated with the normalization

$$
\left(B_{r}^{(2)}+B_{r}^{(3)}\right)\left(\frac{T_{R}}{10^{8} \mathrm{GeV}}\right)\left(\frac{2 \mathcal{M}_{R}}{m_{\phi}}\right)=1 .
$$

One can see that the analytic estimation explains the numerical result quite well and that the required amount of the baryon asymmetry can be easily obtained in the democratic model with the natural scale of perturbations. Another interesting prediction can be seen from the upper-right figure. The amplitude of $\mathrm{U}_{e 3}$ is accurately predicted as $\left|\mathrm{U}_{e 3}\right| \simeq 0.049$, as denoted in the first part of this letter.

In Fig. 2, we show the histogram of the the $n_{B} / s,\left|\mathrm{U}_{e 3}\right|$ and $\mathcal{M}_{\nu}$ for the LOW solution.

\footnotetext{
${ }^{8}$ In the $\mathrm{MSSM},\langle H\rangle=174 \mathrm{GeV} \times \sin \beta$, where $\tan \beta=\langle H\rangle /\left\langle H^{\prime}\right\rangle$ and $H^{\prime}$ is the Higgs field which couples to the down-type quarks (and charged leptons). In Eq. (30), we have taken $\sin \beta \simeq 1$.
} 
In this case, we take the range of perturbations as follows:

$$
\begin{array}{ll}
\delta_{D}=(0.25-1.5) \times 10^{-1}, & \varepsilon_{D}=\delta_{D} 10^{-(3.7-5.8)}, \\
\kappa_{D}=\varepsilon_{D} 10^{-(0-0.7)} e^{i(0-2) \pi}, & \kappa_{R}=\left|\kappa_{D}\right| e^{i\left(-\frac{1}{2} \sim \frac{1}{2}\right) \pi} .
\end{array}
$$

Other conventions are the same as those in Fig 11. The resultant baryon asymmetry is almost the same as in the LMA solution, which is easily understood from the Eq. (30). In the case of the LOW solution, the off diagonal elements in the light neutrino mass matrix are much smaller than those in the LMA solution $\kappa_{\nu} \sim \varepsilon_{\nu} \sim \delta_{\nu} \times 10^{-(4-6)}$, which results in the much more precise prediction on the amplitude of the $\mathrm{U}_{e 3}$.

\section{Conclusions}

In this letter we have investigated leptogenesis with almost degenerate neutrinos, in the framework of democratic mass matrix, which explains very successfully the observed large mixings of the neutrinos as well as quark masses and mixings. The almost degenerate Majorana neutrinos with masses of order $m_{i}^{\nu} \sim \mathcal{O}(0.1) \mathrm{eV}$ induce a considerable rate of the neutrinoless double beta decays, which is accessible in near future experiments. We have shown that the empirical baryon asymmetry is well explained by the decays of the right-handed neutrinos produced in the inflaton decay.

In this model, the $\mathrm{U}_{e 3}$ component of the mixing matrix for neutrino oscillations is predicted as $\left|\mathrm{U}_{e 3}\right| \simeq 0.05$, which is a direct consequence of the nearly $\mathrm{S}_{3}$ symmetric form of the neutrino mass matrix. Such a value of $\mathrm{U}_{e 3}$ will be also tested in the long baseline experiments, such as JHF [18].

\section{Appendix}

In this appendix, we show that if the light neutrinos are degenerate in masses of $m_{i}^{\nu} \simeq \mathcal{O}(0.1) \mathrm{eV}$, the produced lepton asymmetry is highly suppressed in the case of the leptogenesis scenario where the right-handed neutrinos are produced by thermal scatterings. We should stress that the following discussion is a generic consequence of the almost degenerate light neutrinos, independent of the specific models.

The wash-out effect of the lepton asymmetry in the leptogenesis by the decays of thermally produced right-handed neutrino $N_{R i}$ crucially depends on the following parameter $K_{i}:$

$$
K_{i} \equiv \frac{\Gamma_{N_{R i}}}{H\left(T=M_{R i}\right)},
$$


where $H\left(T=M_{R i}\right)$ is the Hubble parameter when the temperature $T$ becomes as low as the mass of the decaying right-handed neutrino, $M_{R i}$. The so-called out-of-equilibrium condition is roughly given by $K_{i} \lesssim 1$, and the final lepton asymmetry is strongly suppressed for $K_{i} \gg 1$ [20]. The parameter $K_{i}$ can be rewritten in terms of a mass parameter $\widetilde{m_{i}}$ [20]:

$$
K_{i} \simeq \frac{\widetilde{m}_{i}}{0.001 \mathrm{eV}}
$$

where

$$
\widetilde{m_{i}} \equiv \sum_{k}\left|\widetilde{h}_{i k}\right|^{2} \frac{\langle H\rangle}{M_{R i}}
$$

We use the Yukawa couplings $\widetilde{h}_{i k}$ defined in the basis where the both mass matrices of right-handed neutrinos and light neutrinos are diagonal:

$$
\sum_{i} \widetilde{h}_{i j} \widetilde{h}_{i k} \frac{\langle H\rangle}{M_{R i}}=m_{j}^{\nu} \delta_{j k}
$$

Let us define a matrix $X_{i j}$ as follows:

$$
X_{i j} \equiv \widetilde{h}_{i j} \frac{\langle H\rangle}{\sqrt{M_{R i} m_{j}^{\nu}}} .
$$

Then from Eq. (37) one can show

$$
\left(X^{T} X\right)_{i j}=\delta_{i j}=\left(X X^{T}\right)_{i j}
$$

Thus, we see that the $\widetilde{m}_{i}$ parameter in Eq. (36) is bounded from below as follows:

$$
\begin{aligned}
\widetilde{m}_{i} & =\sum_{k} m_{k}^{\nu}\left|X_{i k}\right|^{2} \\
& >\min _{j}\left\{m_{j}^{\nu}\right\} \sum_{k}\left|X_{i k}\right|^{2} \\
& \geq \min _{j}\left\{m_{j}^{\nu}\right\}\left|\sum_{k} X_{i k}^{2}\right| \\
& =\min _{j}\left\{m_{j}^{\nu}\right\} .
\end{aligned}
$$

In the last equation, we have used Eq. (39). Therefore, if the light neutrinos are degenerate as $m_{1}^{\nu} \simeq m_{2}^{\nu} \simeq m_{3}^{\nu} \simeq \mathcal{O}(0.1) \mathrm{eV}$, the $K$ parameter becomes at least as large as $K>\mathcal{O}(100)$ [see Eq. (35)], which leads to a strong suppression of the lepton asymmetry generated by the decays of thermally produced right-handed neutrinos. 


\section{References}

[1] H. Harari, H. Haut and J. Weyers, Phys. Lett. B 78 (1978) 459.

[2] Y. Koide, Phys. Rev. D 28 (1983) 252; Phys. Rev. D 39 (1989) 1391.

[3] M. Fukugita, M. Tanimoto and T. Yanagida, Phys. Rev. D 57 (1998) 4429 arXiv:hepph/9709388.

[4] H. Fritzsch and Z. Z. Xing, Phys. Lett. B 440 (1998) 313 [arXiv:hep-ph/9808272].

[5] H. Fritzsch and Z. Z. Xing, Phys. Lett. B 372 (1996) 265 [arXiv:hep-ph/9509389].

[6] M. Apollonio et al., Phys. Lett. B466 (1999) 415 arXiv:hep-ex/9907037.

[7] Y. Fukuda et al. [Super-Kamiokande Collaboration], Phys. Lett. B 433 (1998) 9 arXiv:hep-ex/9803006;

Phys. Lett. B 436 (1998) 33 arXiv:hep-ex/9805006;

Phys. Rev. Lett. 81 (1998) 1562 arXiv:hep-ex/9807003.

See also a recent data, C. Yanagisawa, Nucl. Phys. Proc. Suppl. 95 (2001) 93.

[8] Y. Fukuda et al. [Super-Kamiokande Collaboration], Phys. Rev. Lett. 81 (1998) 1158 [Erratum-ibid. 81 (1998) 4279] [arXiv:hepex/9805021; ;

Phys. Rev. Lett. 82 (1999) 2430 arXiv:hep-ex/9812011;

Phys. Rev. Lett. 82 (1999) 1810 arXiv:hep-ex/9812009.

[9] H. V. Klapdor-Kleingrothaus et al. [GENIUS Collaboration], arXiv:hep-ph/9910205.

[10] E. Fiorini, Phys. Rept. 307 (1998) 309; Nucl. Phys. Proc. Suppl. 100 (2001) 332.

[11] H. Ejiri, J. Engel, R. Hazama, P. Krastev, N. Kudomi and R. G. Robertson, Phys. Rev. Lett. 85 (2000) 2917 arXiv:nucl-ex/9911008.

[12] S. Moriyama, Talk at International Workshop on Technology and Application of Xenon Detectors (Xenon01), ICRR, Kashiwa, Japan, December 3-4, 2001.

[13] S. Waldman, Talk at International Workshop on Technology and Application of Xenon Detectors (Xenon01), ICRR, Kashiwa, Japan, December 3-4, 2001.

[14] M. Fukugita and T. Yanagida, Phys. Lett. B174 (1986) 45.

[15] K. Kumekawa, T. Moroi and T. Yanagida, Prog. Theor. Phys. 92 (1994) 437 arXiv:hep-ph/9405337; 
G. Lazarides, Springer Tracts Mod. Phys. 163 (2000) 227 arXiv:hep-ph/9904428 and references therein;

G. F. Giudice, M. Peloso, A. Riotto and I. Tkachev, JHEP 9908 (1999) 014 [arXiv:hep-ph/9905242];

T. Asaka, K. Hamaguchi, M. Kawasaki and T. Yanagida, Phys. Lett. B 464 (1999) 12 arXiv:hep-ph/9906366, Phys. Rev. D 61 (2000) 083512 arXiv:hep-ph/9907559].

[16] G. L. Fogli, E. Lisi, D. Montanino and A. Palazzo, Phys. Rev. D 64 (2001) 093007 arXiv:hep-ph/0106247.

G. L. Fogli, E. Lisi, A. Marrone, D. Montanino and A. Palazzo, arXiv:hepph/0201290, and references therein.

[17] H. V. Klapdor-Kleingrothaus, A. Dietz, H. L. Harney and I. V. Krivosheina, Mod. Phys. Lett. A 16 (2002) 2409 arXiv:hep-ph/0201231.

[18] Y. Itow et al., arXiv:hep-ex/0106019.

See also http://neutrino.kek.jp/jhfnu/.

[19] T. Yanagida, in Proceedings of the "Workshop on the Unified Theory and the Baryon Number in the Universe", Tsukuba, Japan, 1979, edited by O. Sawada and A. Sugamoto, KEK Report No. KEK-79-18, p. 95; Prog. Theor. Phys. 64 (1980) 1103;

M. Gell-Mann, P. Ramond and R. Slansky, in "Supergravity", edited by D.Z. Freedman and P. van Nieuwenhuizen (North-Holland, Amsterdam, 1979).

[20] For reviews and references, see, for example,

M. Plumacher, Nucl. Phys. B 530 (1998) 207 arXiv:hep-ph/9704231;

W. Buchmuller and M. Plumacher, Phys. Rept. 320 (1999) 329 arXiv:hepph/9904310];

W. Buchmuller and M. Plumacher, Int. J. Mod. Phys. A 15 (2000) 5047 arXiv:hep$\mathrm{ph} / 0007176$.

[21] M. Flanz, E. A. Paschos and U. Sarkar, Phys. Lett. B 345 (1995) 248 [Erratum-ibid. B 384 (1995) 487] arXiv:hep-ph/9411366;

L. Covi, E. Roulet and F. Vissani, Phys. Lett. B 384 (1996) 169 arXiv:hepph/9605319];

W. Buchmuller and M. Plumacher, Phys. Lett. B 431 (1998) 354 arXiv:hepph/9710460].

[22] L. Covi, E. Roulet and F. Vissani, in Ref. 21]. 
[23] For a discussion and references, see A. Pilaftsis, Int. J. Mod. Phys. A 14 (1999) 1811 arXiv:hep-ph/9812256].

[24] V. A. Kuzmin, V. A. Rubakov and M. E. Shaposhnikov, Phys. Lett. B 155 (1985) 36.

[25] S. Y. Khlebnikov and M. E. Shaposhnikov, Nucl. Phys. B308 (1988) 885;

J. A. Harvey and M. S. Turner, Phys. Rev. D 42 (1990) 3344.

[26] D. E. Groom et al. [Particle Data Group Collaboration], "Review of particle physics," Eur. Phys. J. C 15 (2000) 1. 

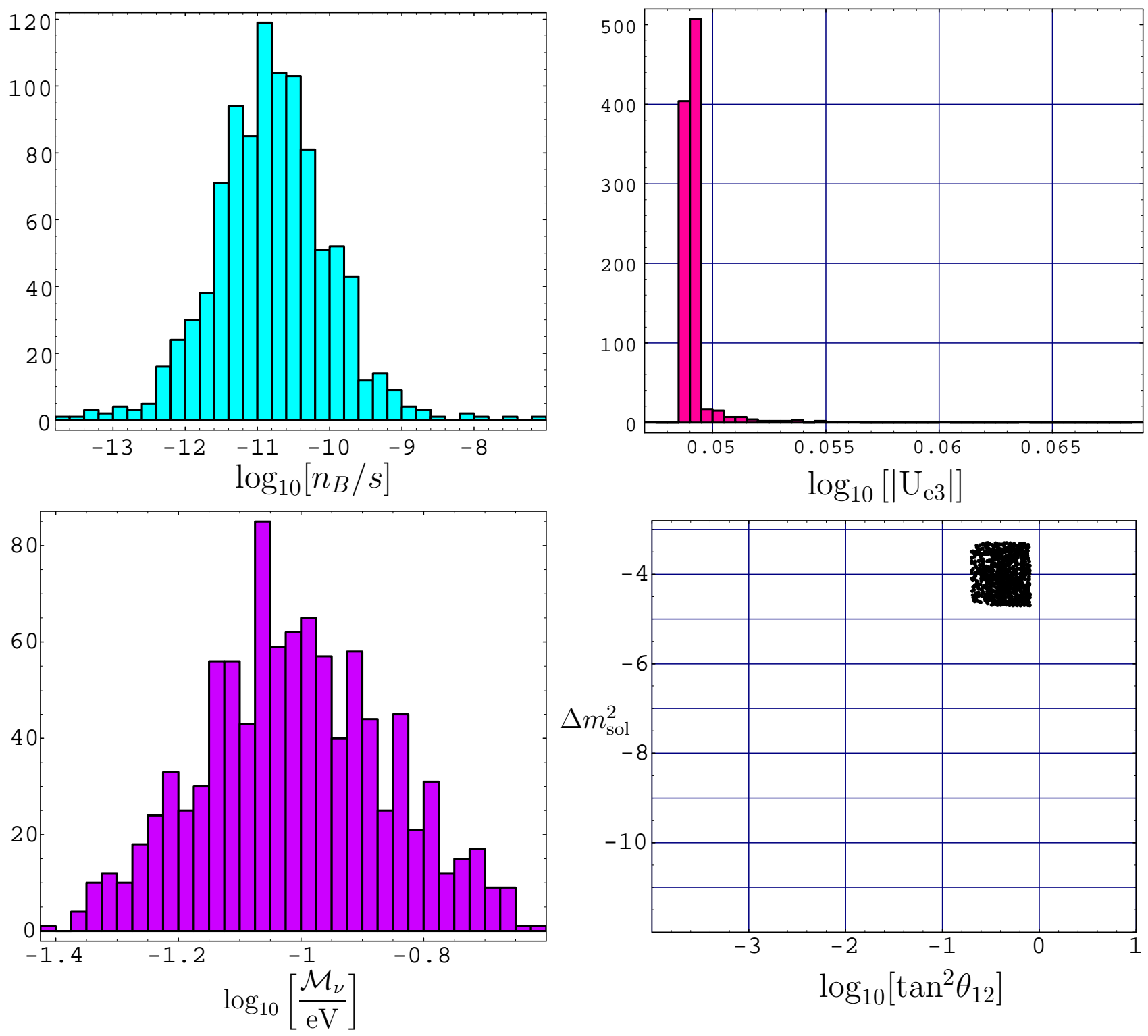

Figure 1: Histograms of the $n_{B} / s,\left|\mathrm{U}_{e 3}\right|$ and $\mathcal{M}_{\nu}$. We randomly generate the small perturbations as in Eq. (31), and collect the data set of these quantities if the generated mass spectrum and mixing angles of the light neutrinos satisfy the conditions for the LMA solution in Eq.(14). Then we show the frequencies of these quantities in the vertical axes. The generated mass spectrum and mixing angles for the solar neutrino oscillation is also plotted in the lower-right figure, in which $\Delta m_{\text {solar }}^{2}$ is plotted with $\log _{10}\left[\Delta m_{\text {solar }}^{2} / \mathrm{eV}^{2}\right]$ unit. 

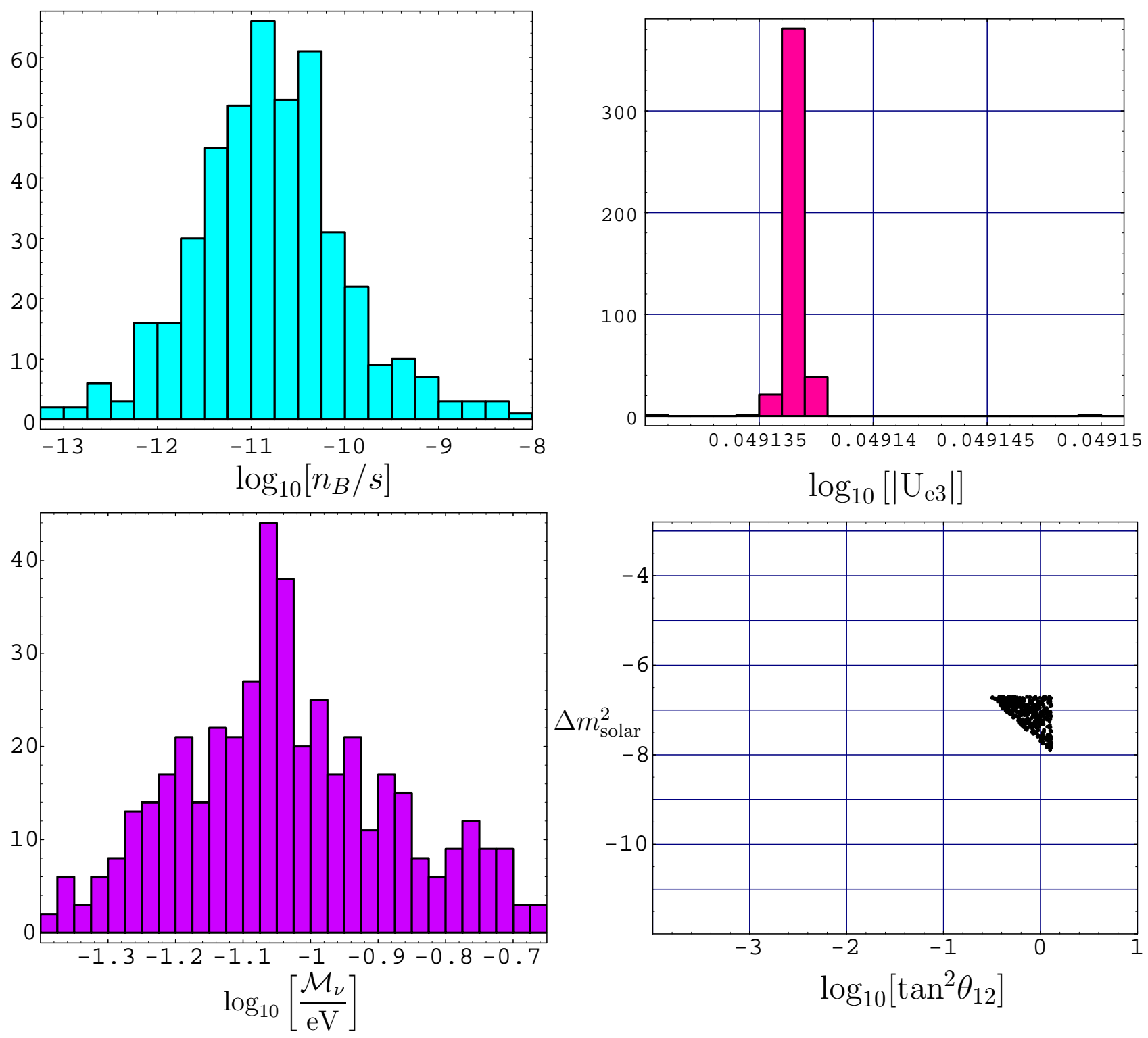

Figure 2: The same as Fig.1, but for the LOW solution. 